\title{
Trends and Treatment Outcomes of Tuberculosis in Debre Berhan Referral Hospital, Debre Drehan, Ethiopia
}

\author{
Tsegahun Asfaw", Moges Lewetegn, Helen Tariku \\ Department of Medical Laboratory Science, Debre Berhan University, Debre Berhan, Ethiopia \\ Email address: \\ tsegahun.asfaw12@gmail.com (T. Asfaw),mogesit@gmail.com (M. Lewetegn), helachina07@gmail.com (H. Tariku) \\ ${ }^{*}$ Corresponding author
}

To cite this article:

Tsegahun Asfaw, Moges Lewetegn, Helen Tariku. Trends and Treatment Outcomes of Tuberculosis in Debre Berhan Referral Hospital, Debre Drehan, Ethiopia. Clinical Medicine Research. Vol. 7, No. 5, 2018, pp. 97-102. doi: 10.11648/j.cmr.20180705.11

Received: August 27, 2018; Accepted: September 10, 2018; Published: October 24, 2018

\begin{abstract}
Tuberculosis (TB) remains major Public health problem and among the top ten leading causes of human deaths worldwide. Moreover, TB is the leading cause of morbidity, one of the three major causes for hospital admission, and the second killer next to malaria in Ethiopia. Early diagnosis, effective treatment and continues assessment of treatment outcome are important to improve TB management program. Retrospective study design was conducted to assess trend and treatment outcomes of TB in Debre Berhan referral hospital. 4 years (2014 to 2017) TB case records was reviewed with standard check list and entered in to EpiData 3.1 and analyzed using SPSS version 16. Among the total registered TB patients with complete record $(\mathrm{n}=506), 256(50.6 \%)$ were males and 250(49.4\%) Female. The treatment cured rate varies from $23.29 \%$ in 2006 E.C to $47.45 \%$ in 2007 E.C, whereas treatment completed varies from $46.91 \%$ in 2007 E.C to $67.08 \%$ in 2009 E.C. Majority of the smear-positive pulmonary tuberculosis patients $183(97.3 \%)$ were cured at the end of their anti-TB treatment. The proportion of HIV co-infection among TB patients was $21.3 \%$. However, overall cure rate was nearly the same in both HIV positive and negative patients. As conclusion majority of the smear-positive pulmonary tuberculosis patients were cured at the end of their anti-TB treatment. There was also a continuous increment of treatment success rate, indicating that the hospital is within the track of WHO target currently. However the proportion of HIV co-infection among TB patients was higher which signifies the urgent need for staff capacity building and increasing public awareness.
\end{abstract}

Keywords: Trends, Treatment Outcomes, Tuberculosis

\section{Background}

Tuberculosis [TB] remains major Public health problem and among the top ten leading causes of human deaths worldwide. In 2015, alone 9.6 million new cases and 1.5 million deaths were reported globally. Under resourced areas, prevalence were high particularly in south East Asian and western pacific regions with proportion of 58\% share from total global estimate of new TB cases; and Africa continent were home for $28 \%$ of the global new TB cases, including the most sever Burdon of TB with 281 cases for every 1000,000 people, which is more than double the global average of 133 cases per 100,000 populations [1].

TB prevalence and incidence reduced substantially globally after implementation of DOTS program. In Ethiopia, Even if case detection rate improved from the base line 30 to
71.8 in 2006/07, TB treatment success rate improved from the base line 85 to 90.6 and TB cure rate from the baseline $69.0 \%$ in $2006 / 07$ to 91 in 2013, and reduce the mortality rate by $50 \%$ [2]. The country Still ranks second in Africa after Nigeria and seventh among the world's 22 high TB burden countries with estimated TB prevalence rate of 227/1000000 population , $18 \mathrm{~TB}$ deaths and with an estimated incidence rate of 224 per 100,000 populations respectively [3]. More over the country ranked 15 th with more than 5000 estimated MDR-TB patients each year among 27 high MDR-TB countries [4].

Several reasons and risk factors for poor TB treatment outcomes have been reported. High age, low income, no or limited access to transport, distance from home to the 
treatment center, incomplete treatment compliance, limited interest in information about the disease and its treatment, limited social support, multidrug resistance, and comorbidity have all been found to be related to unsuccessful treatment outcomes [5]. Therefore, this study is designed to assess trend of TB and treatment outcomes in Debre Berhan referral hospital.

\section{Material and Methods}

\subsection{Study Area and Period}

This study was conducted from Jan 2014- Jan 2018 in Debre Berhan referral hospital which is $130 \mathrm{kms}$ from Addis Ababa.

\subsection{Study Design}

Retrospective study design was conducted.

\subsection{Sample Size}

All patients presumptive for TB (pulmonary or extra pulmonary) and who had full documentation during the study period were included but Patients who had incomplete data, e.g., age, sex Gene-X-pert results were excluded from the study. Between January 2014 and January 2018, a total of 537 tuberculosis patients were registered. Of these, 506 (94.22\%) tuberculosis cases had complete records.

\subsection{Operational Definitions}

Extra pulmonary tuberculosis (EPTB): a case of TB that involves organs other than the lung e.g. pleura, lymph nodes, abdomen, genitourinary tract, skin, joints, bones and meninges.

Pulmonary tuberculosis (PTB): a case of TB involving the lung parenchyma.

Pulmonary TB, smear positive (SPPTB): SPPTB case is based on the presence of at least one acid fast bacillus $(\mathrm{AFB}+)$ in at least one sputum sample in countries with a well-functioning EQA system. In countries without functional EQA, a SPPTB case is defined as one with two or more initial sputum smear examinations positive for AFB, or one sputum smear examination positive for AFB plus radiographic abnormalities consistent with active PTB as determined by a clinician or one sputum smear positive for AFB plus sputum culture-positive.

Pulmonary TB, smear-negative (SNPTB): a case of PTB is considered to be smear-negative if at least two sputum specimens at the start of treatment are negative for AFB in countries with a functional EQA system.

Cured: defined as patients whose sputum smear or culture was positive at the beginning of the treatment but smear or culture negative in the last month of treatment and on at least one previous occasion.

Treatment completed: interpreted as patients who completed treatment without evidence of failure but did not have a negative sputum smear or culture result in the last month of treatment and on at least one previous occasion.

Treatment defaulter: defined as was a patient who had been on treatment for at least four weeks and whose treatment was interrupted for eight or more consecutive weeks.

Treatment failure: defined as patients whose sputum smear or culture was positive at the fifth month or later during treatment.

Transfer out: defined as a patient who had been transferred to another recording and reporting unit and whose treatment outcome was unknown at the original registering unit.

\subsection{Data Collection}

Structured checklist was used to collect demographic data and clinical information from TB registration book with trained data collectors.

\subsection{Data Processing and Analysis}

The data was collected from registration book with trained person with standard check list. Cleaning and analyses was done using EpiData 3.1 and SPSS software version 21. A double data entry was implemented to avoid data entry errors. Together with demographic and socio economic factors was tabulated in to frequency table to see possibility of risk association with the dependent outcome variable. Univariate or multivariate analysis was used. OR within $95 \%$ C.I. and P-Value Less than 0.05 was considered as significant association.

\subsection{Ethical Considerations}

An ethical approval was obtained from Debre Berhan University ethical review and research committee. Permission was obtained from administration of Debre Berhan referral hospital, where the study was carried out.

\section{Result}

\subsection{Socio-Demographic Characteristics}

Among the total registered TB patients with complete record $(n=506), 256(50.6 \%)$ were males and $250(49.4 \%)$ Female. The median age of patients was 30 years, six in ten of the study participants were children aged less than 15 years; near to seven in ten were adults with ages ranging from 15 to 44 years; Three in ten $(33.4 \%)$ were smear positive PTB, nearly three fifth (54.9\%) were smear negative PTB and the rest were EPTB patients. 473(93.7\%) were new, $25(4.9 \%)$ were relapse, retreatment was $2(0.4 \%)$, treatment failure was $2(0.4 \%)$ and other cases were $3(0.6 \%)$. Eight in ten $78.7 \%$ (398) were HIV negative and 2 in ten which is $21.3 \%$ (108) were HIV positive (Table 1). 
Table 1. Socio-demographic and disease related characteristics of registered TB patients (n=506) in Debre Berhan referral Hospital.

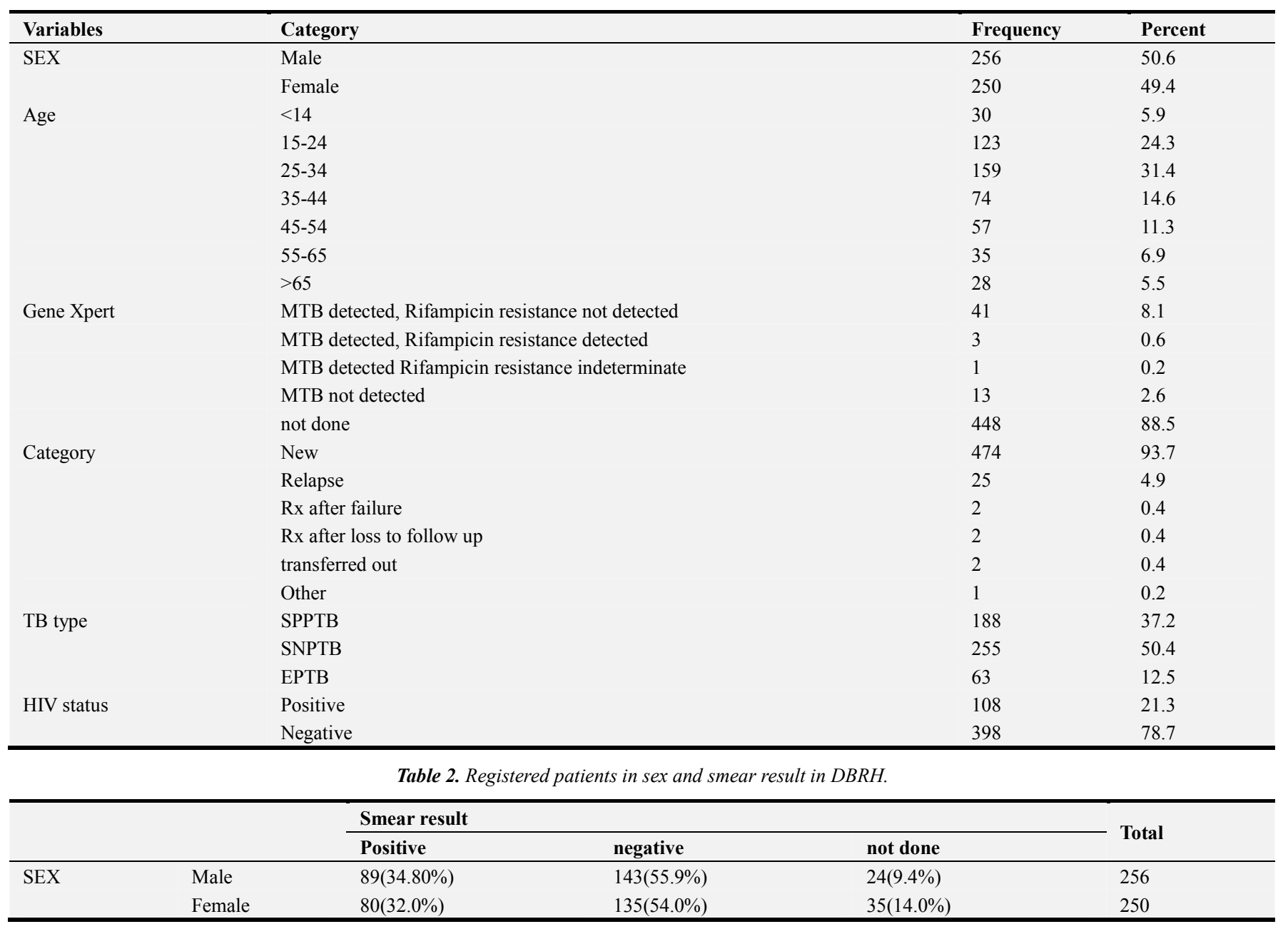

\subsection{Trend of TB in Deber Berhan Referral Hospital from 2014-2017}

The treatment cured rate varies from $23.29 \%$ in 2006 E.C to $47.45 \%$ in 2007 E.C, whereas treatment completed varies from $46.91 \%$ in 2007 E.C to $67.08 \%$ in 2009 E.C (figure 1).

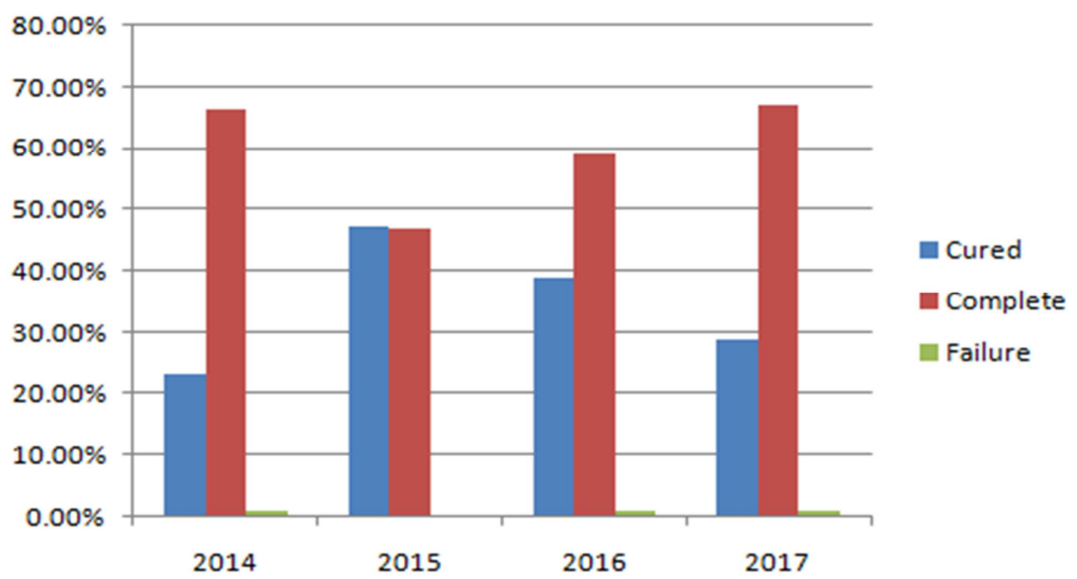

Figure 1. Trend of treatment outcome of TB patients in DBRH, 2014-2017.

\subsection{Treatment Outcomes}

From the total of 506 registered tuberculosis patients included in the study, 480 (94.9\%) had successful treatment outcome (favorable treatment outcome). Of these, $172(34 \%)$ and $308(60.9 \%)$ patients were cured and treatment completed, respectively. Similarly $10(2 \%), 2(0.4 \%), 9$ $(1.8 \%)$ and $4(0.8 \%)$ were transferred out, defaulted, dead and treatment failed, respectively (able 3). 
Table 3. Treatment outcomes of all TB patients in DBRH.

\begin{tabular}{llll}
\hline & & Frequency & Percent \% \\
\hline Treatment out come & Cured & 172 & 34 \\
& Rx completed & 308 & 60.9 \\
& Failure & 4 & 0.8 \\
& Death & 9 & 1.8 \\
& Loss & 1 & 0.2 \\
& transfered out & 10 & 2 \\
& not evaluated & 1 & 0.2 \\
& MDR TB & 1 & 0.2 \\
\hline
\end{tabular}

\subsection{Distribution of Age with TB Type}

Among TB patients with full record, aged 25-34 had high percentage (33.5\%) of SPPTB, and EPTB (34.9\%). Low percentage of SPPTB $(2.7 \%)$ and EPTB $(1.6 \%)$ were in patients with age group of $<14$ and $>65$. Elevated and decreased percent of SNTB were exhibited in age groups of $25-34(29 \%)$ and 55-65 (6.7\%) respectively (figure 2).

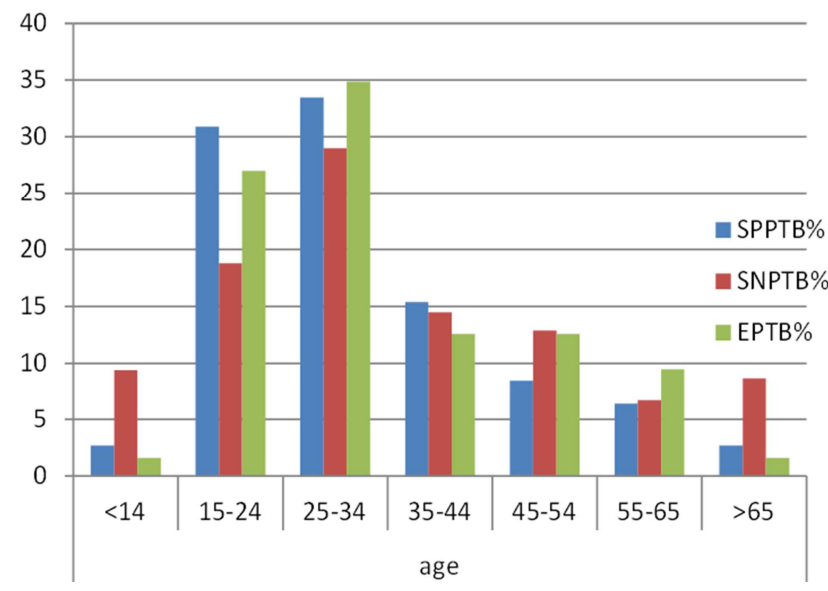

Figure 2. TB type in percentage with age categories in DBRH.

\subsection{Outcomes of Treatment in HIV Patients}

Overall cure rate was nearly the same in both HIV positive and negative patients. Death rate was higher in positive HIV patients than negatives. Treatment completed \& failure rate are almost comparable (figure 3 ).

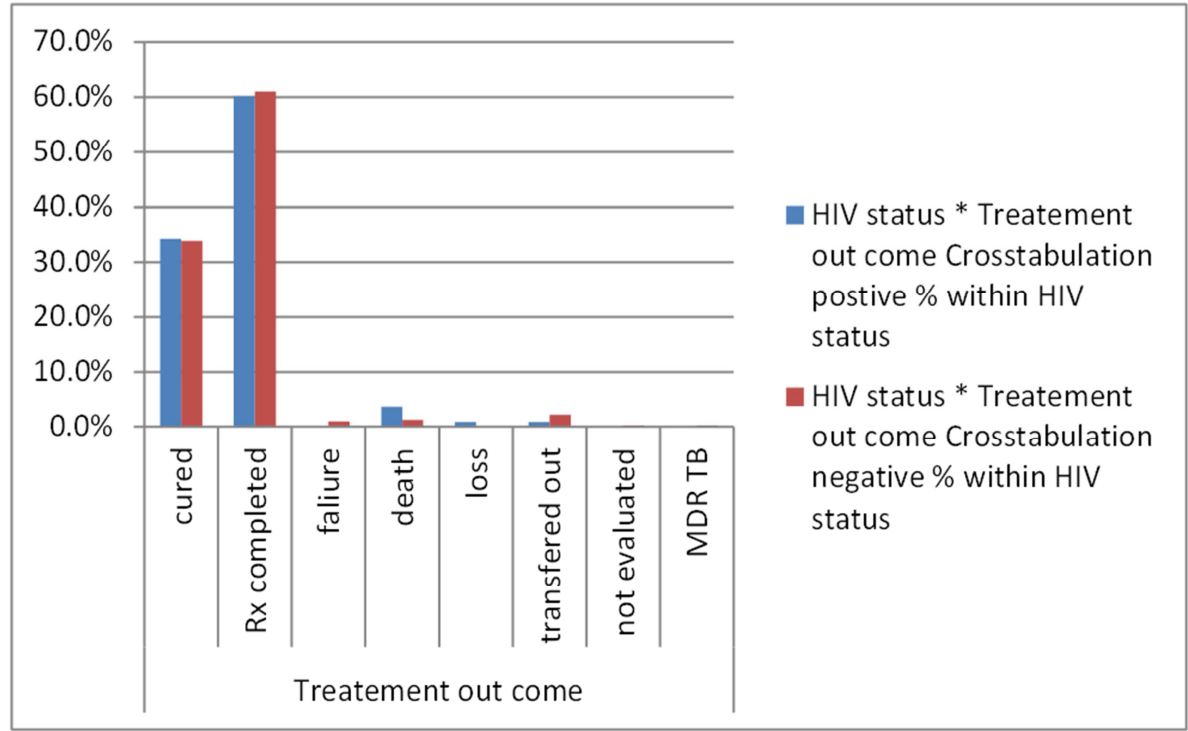

Figure 3. Treatment outcomes with HIV patients in DBRH.

Among male participants 240 (93.8\%) were showed favorable treatment outcomes and 16 (6.2\%) showed unfavorable treatment outcomes while female showed 240
(96\%) favorable outcomes. From participants that undergo GeneXpert test $40(97.6 \%)$ showed favorable treatment outcomes (Table 4).

Table 4. Distribution of treatment outcomes of registered TB patients $(n=506)$ by socio-demographic and disease characteristics in Debre Berhan Hospital.

\begin{tabular}{lllll}
\hline \multirow{2}{*}{ Variable } & Categories & Treatment outcome & \\
\cline { 3 - 5 } & & Favorable outcome N (\%) & Unfavorable outcome N (\%) & Total \\
\hline Sex & Male & $240(93.8)$ & $16(6.2)$ & 256 \\
& Female & $240(96)$ & $10(4)$ & 250 \\
Age & $0-14$ & $30(100)$ & $0(0 \%)$ & $3(4.1)$ \\
& $15-24$ & $118(95.9)$ & $5(3.2)$ & 123 \\
& $25-34$ & $154(96.8)$ & $2(2.7)$ & 74 \\
& $35-44$ & $72(97.3)$ & $8(14)$ & 57 \\
& $45-54$ & $49(86)$ & $2(5.7)$ & 35 \\
Gene Xpert & $55-65$ & $33(94.3)$ & $4(14.3)$ & 28 \\
\hline
\end{tabular}




\begin{tabular}{|c|c|c|c|c|}
\hline \multirow{2}{*}{ Variable } & \multirow{2}{*}{ Categories } & \multicolumn{2}{|l|}{ Treatment outcome } & \multirow{2}{*}{ Total } \\
\hline & & Favorable outcome N (\%) & Unfavorable outcome N (\%) & \\
\hline \multirow{9}{*}{ Category } & MTB detected, Rifampicin resistance detected & $3(100)$ & $0(0)$ & 3 \\
\hline & MTB detected, Rifampicin Resistance indeterminate & $1(100)$ & $0(0)$ & 1 \\
\hline & MTB not Detected & $13(100)$ & $0(0)$ & 13 \\
\hline & Not done & $423(94.4)$ & $25(5.6)$ & 448 \\
\hline & New & $451(95.2)$ & $23(4.8)$ & 474 \\
\hline & Relapse & 24(96) & $1(4)$ & 25 \\
\hline & Rx failure & $2(100)$ & $0(0)$ & 2 \\
\hline & $\mathrm{Rx}$ after loss to follow up & $1(50)$ & $1(50)$ & 2 \\
\hline & Transferred out & $2(100)$ & $0(0)$ & 2 \\
\hline \multirow{3}{*}{ TB type } & Other & $0(0)$ & $1(100)$ & 1 \\
\hline & SPPTB & $183(97.3)$ & $5(2.7)$ & 188 \\
\hline & SNPTB & $237(92.9)$ & $18(7.1)$ & 255 \\
\hline \multirow{3}{*}{ HIV status } & ЕРТВ & $60(95.3)$ & $3(4.7)$ & 63 \\
\hline & Positive & $102(94.5)$ & $6(5.5)$ & 108 \\
\hline & Negative & $378(95)$ & $20(5)$ & 398 \\
\hline
\end{tabular}

\section{Discussion}

Among 506 TB patients registered in DOTS clinic in the DebreBerhan referral hospital, almost half of the patients are smear negative pulmonary tuberculosis 255 (50.4\%); where as two out of five patients $(37.2 \%)$ are smear positive pulmonary tuberculosis and the remaining are extra pulmonary TB $63(12.5 \%)$. Majority of the smear-positive pulmonary tuberculosis patients $183(97.3 \%)$ were cured at the end of their anti-TB treatment. It is comparable with reports from study done at Tigray region, Ethiopia (85.5\%) [6]

The high proportion of SPPTB observed among male TB patients in this study was consistent with the study conducted in Gambella regional hospital [7] and the epidemiological analysis of TB trends in Ethiopia [8], in which men are disproportionately affected by TB.

According to this study, there was a continuous increment of treatment success rate from the end of 2014 to 2017 . The treatment success rate between 2014 and 2017 was $89.7 \%$, $94.4 \%, 98.0 \%, 96.1 \%$ in respective years and overall was $94.9 \%$, which is better than the WHO international target of $87 \%$ [9]. This might be a clue indicating that the hospital is within the track of WHO target currently.

The majority of TB patients, 480 (94.9\%), had successful treatment outcome (cured + treatment completed) in comparison with other hospitals like Gondar University Hospital (29.5\%) [10] Felege Hiwot Referral Hospital (26\%) [11], Gambo Rural Hospital (66.9\%) [12]. It also improved from the past 5 years prior to study period which was $79.4 \%$. The present study showed successful treatment outcome (94.9\%) which met the target (90\%) set in the Global Plan to stop TB by 2015 [13]. The average cure rate of tuberculosis was $34 \%$, almost similar with a study done in Gambella, Ethiopia (30.7\%) [14]. This shows the hospital is doing well in comparison.

The average treatment completed rate was $60.9 \%$ in this study, which was in agreement with $64.6 \%$ according to study done in Addis Ababa, Ethiopia [15]. However, it was higher than $9.5 \%$ in Gondar Hospital, Northwest Ethiopia [10], and 25\% in Gambella, Ethiopia [7]. The default rate in this study $1(0.2 \%)$ was also lower than the average $6.2 \%$ observed among other studies conducted in the country; $22.9 \%, 18.3 \%$ and $2.5 \%$ in Gambella, Gondar and Felege Hiwot hospitals, respectively [7, 10, 11]. This lower defaulter rate in this study might be due to proper supervision and health education in the study area.

The TB treatment failure rate of this study was $4(0.8 \%)$, which was lower than the average TB treatment failure rates that ranged from $0.2 \%$ to $18.6 \%$; reported from the different parts of Ethiopia [10, 16]. In addition, $1.8 \%$ of the TB patients died between 2014 and 2017, which was relatively consistent with an average of 3.7\% in Addis Ababa, Ethiopia [15]. However it was lower than $10.1 \%$ in Gondar Hospital, Northwest Ethiopia [16].

In this study, the proportion of HIV co-infection among TB patients was $21.3 \%$; nearly similar to the studies conducted in Nigeria (20.0\%) [17]. However, this finding is lower than that of study conducted in Northwest Ethiopia (25\%) [10], and higher than that of 2013 WHO report for Ethiopia (9.6\%). This high prevalence of HIV co-infection among TB patients in the study area signifies the urgent need for staff Capacity building and increasing public awareness.

\section{Conclusion and Recommendation}

In this study, majority of the smear-positive pulmonary tuberculosis patients $183(97.3 \%)$ were cured at the end of their anti-TB treatment. There was a continuous increment of treatment success rate from the end of 2014 to 2017 in the hospital. The treatment success rate in the year 2014-2017 was $94.9 \%$, indicating that the hospital is within the track of WHO target currently. The proportion of HIV co-infection among TB patients was $21.3 \%$, which is higher than that of 2013 WHO report for Ethiopia (9.6\%). This high prevalence of HIV co-infection among TB patients in the study area signifies the urgent need for staff Capacity building and increasing public awareness. And also TB prevention and control should be strengthened, data record keeping system, including patient information should be improved.

\section{Conflicting of Interests}

The authors declare that they have no conflict of interests. 


\section{Funding}

This research is funded by Debre Berhan University

\section{Authors' Contributions}

TA and HT - Collect and analyzed the data. TA - Analyze the data and wrote the manuscript. All authors read and approved the final manuscript.

\section{Acknowledgements}

We would like to thank Debre Berhan University for giving this opportunity. We also thank laboratory and nurse staffs of Debre Berhan referral hospital for their help during data collection process.

\section{References}

[1] World Health Organization, Global Tuberculosis Control: WHO Report 2015, WHO/HTM/TB/ 2015. 22, World Health Organization, Geneva, Switzerland, 2015.

[2] World Health Organization, Global Tuberculosis Control report: WHO Report 2014, WHO/HTM/TB/ 2014. 08, World Health Organization, Geneva, Switzerland, 2014.

[3] World Health Organization, Global Tuberculosis Control: WHO Report 2011, WHO/HTM/TB/ 2011.16, World Health Organization, Geneva, Switzerland, 2011.

[4] Federal Democratic Republic of Ethiopia, Ministry of Health (FMOH) (2009) TB/ HIV implementation guideline, Addis Ababa, Ethiopia.

[5] Ai X, Men K, Guo L et al., Factors associated with low cure rate of tuberculosis in remote poor areas of Shaanxi Province, China: a case control study, BMC Public Health, 2010 vol. 10, article 112 .

[6] Berhe G, Enquselassie F, and Aseffa A, Treatment outcome of smear-positive pulmonary tuberculosis patients in Tigray Region, Northern Ethiopia, BMC Public Health, 2012, vol. 12, p. 537.

[7] Demeke D, Legesse M, Bati J. Trend of Tuberculosis and
Treatment Outcomes in Gambella Region with Special Emphasize on Gambella Regional Hospital, Western Ethiopia. J Mycobac Dis, 2013, 3: 130.

[8] Nigatu T, Abraha M (2010) Epidemiological analysis of tuberculosis trends in Ethiopia Tüberküloz, 2010; 58: 375384.

[9] World Health Organization: Global Plan to Stop TB 20112015. Davos, Switzerland: WHO; 2006.

[10] Tessema B, Muche A, Bekele A, Reissig D, Emmrich F, and Sack U. Treatment outcome of tuberculosis patients at Gondar University Teaching Hospital, Northwest Ethiopia. A five year retrospective study, BMC Public Health, 2009; vol. 9, article 371.

[11] Biadglegne F, Anagaw B, Debebe T et al., A retrospective study on the outcomes of tuberculosis treatment in Felege Hiwot Referral Hospital, Northwest Ethiopia, International Journal of Medical Sciences, 2013; vol. 5, no. 2, pp. 85-91.

[12] Ramos JM, Reyes F, and Tesfamariam A, Childhood and adult tuberculosis in a rural hospital in Southeast Ethiopia: a tenyear retrospective study, BMC Public Health, 2010; vol. 10, article 215 .

[13] World Health Organization, G Global Tuberculosis Report 2012, WHO, Geneva, Switzerland, 2012.

[14] Sisay S, Mengistu B, Erku W, WoldeyohannesD. Directly Observed Treatment Short-course (DOTS) for tuberculosis control program in Gambella Regional State, Ethiopia: ten years' experience. BMC Res Notes, 2014;7(44):1756-0500.

[15] Belete G, Gobena A, Girmay M, Sibhatu B. Treatment outcome of tuberculosis patients under directly observed treatment in Addis Ababa, Ethiopia. Braz J Infect Dis, 2013;17(5):521-528.

[16] Belay T, Abebe M, Assegedech B, Dieter R, Frank E, Ulrich S. Treatment outcome of tuberculosis patients at Gondar University Teaching Hospital, Northwest Ethiopia: Afive-year retrospective study. BMC Public Health, 2009; 9(371):14712458.

[17] Ukwaja KN, Modebe O, Igwenyi C, Alobu I. The economic burden of tuberculosis care for patients and households in Africa: a systematic review. The International Journal of Tuberculosis and Lung Disease, 2012; 16(6); 733-739. 